\title{
Development of Rate Gyroscope Characterization Tools with Application to Helium Exposure Testing
}

\author{
Evan W. Hilgemann, ${ }^{*}$ Hunter S. Ruch, ${ }^{\dagger}$ and James W. Cutler ${ }^{\ddagger}$ \\ Department of Aerospace Engineering, University of Michigan, Ann Arbor, Michigan 48109
}

\begin{abstract}
The purpose of this paper is to develop procedures for determining the characteristics of a rate gyroscope and apply them to units that have been exposed to helium. However, the developed methods are also relevant to other forms of inertial sensors. The work is motivated by the importance of inertial measurement devices, and knowledge of their calibration parameters, to spacecraft attitude determination problems. Procedures to measure these parameters are discussed and a visualization tool used to track scale factor and bias errors over time is established. The unique contribution of this paper is to characterize how exposure to helium affects rate gyroscope measurements. Although the negative effects of helium have been known for some time, there has been little effort to characterize how a complete unit reacts. This characterization is important due to the potential for a spacecraft to be exposed to helium on launch vehicles. Three separate sensors, a muRata SCR1100 single axis gyro, an ST L3G4200D three axis gyro, and an Epson M-G350-PD11 IMU are exposed to helium. The calibration parameters of these units are then tracked over time. All of these devices have spaceflight heritage on Michigan Exploration Laboratory satellites or will be flown on an upcoming mission. The first two units exhibited varying levels of undesired behavior after helium exposure, while the Epson remained unaltered. Additionally, a method to protect the units using epoxy encapsulation is proposed and tested on two L3G4200D sensors. Even though the encapsulation appears to have negated helium related issues, the epoxy application process itself resulted in other undesired side effects.
\end{abstract}

\section{Introduction}

The purpose of this report is to develop characterization tools for MEMS sensors, particularly rate gyroscopes, and apply the tools to units that have been exposed to helium. A basic model for a rate gyroscope is discussed and a method to visualize change in calibration parameters over time is established. Furthermore, angular random walk (ARW) is calculated using the Allan variance method and applied to measure noise parameters. These tools are used to analyze the response of various MEMS devices to a helium environment. This information is relevant due to the importance of inertial sensors in spacecraft attitude determination and control and the potential for these sensors to be negatively impacted by exposure to helium aboard a launch vehicle. Various studies have measured diffusion of helium through MEMS packaging ${ }^{1}$ and change in sensor resonator behavior after exposure to helium. ${ }^{2,3}$ However, the response of system level units to helium has not been well categorized. The hazard posed to spacecraft was originally noticed by the Aerospace Corporation following their AeroCube missions. ${ }^{4}$ Similar problems were observed on Michigan Exploration Laboratory's (MXL) MCubed-2 mission. ${ }^{5}$

Testing was performed on three inertial measurement models, a muRata SCR1100 single axis gyro, ${ }^{6}$ an ST L3G4200D 3-axis gyro, ${ }^{7}$ and an Epson M-G350-PD11 IMU. ${ }^{8}$ The SCR1100 unit is identical to the one flown on MCubed-2, while the L3G will be incorporated into future spacecraft in development by MXL. Finally, the Epson unit will be flown on an upcoming MXL CubeSat mission named CADRE. ${ }^{9}$ In addition to gyro measurements, the accelerometer measurements from the Epson are also analyzed. All three units

*MSE Student. Email: ehilge@umich.edu

${ }^{\dagger}$ BSE Student. Email: hsruch@umich.edu

${ }^{\ddagger}$ Professor. Email: jwcutler@umich.edu 
were exposed to high helium concentrations for up to 24 hours, an amount of time similar to what could be experienced on a launch vehicle. The performance of each unit was then tracked over 1-5 months. Additionally, two L3G units were encased in epoxy before being exposed to helium with the goal of verifying if the epoxy could act as an effective barrier to helium diffusion.

The analysis methods used to characterize the units are described in Section II. Basic procedures and results for the helium soak experiments are presented in Section III while the epoxy encapsulation experiment is discussed separately in Section IV.

\section{Analysis Tools}

This section focuses on the development of an appropriate sensor model, followed by a description of the parameters calculated to characterize the sensors.

\section{A. Sensor Model}

A simple model for a gyroscope is:

$$
\tilde{\omega}(t)=\alpha \cdot \omega(t)+\beta+\eta,
$$

where $\tilde{\omega}$ is the measured angular rate, $\omega$ is the actual angular rate, $\alpha$ and $\beta$ are parameters corresponding to scale factor and slowly varying zero offset bias, and $\eta$ refers to inherent noise in the system. Note that this equation does not explicitly include other terms such as axis misalignment which cannot be distinguished from scale factor error given the test conditions. However, previous experiments done to calculate similar parameters as part of the RAX CubeSat mission have shown that a misalignment error of $15^{\circ}$ resulted in a calculated scale factor error of only $3.5 \%$. If the misalignment error is reduced to $5^{\circ}$, the error is only $0.3 \%{ }^{10}$ Since the effects are relatively small, it is assumed that misalignment is captured by the $\eta$ term.

\section{B. Visualization of Bias and Scale Factor Parameters}

One can experimentally determine scale factor and bias by rotating a gyro about an axis of interest at $n$ different angular velocities, denoted as a vector $\vec{\omega}$, for some amount of time. The $i^{t h}$ estimated angular velocity, $\tilde{\omega}_{i}$, is then the mean measurement of $\omega_{i}$ and has a standard deviation $\sigma_{i}$,

$$
\vec{\omega}_{\text {true }}=\left[\begin{array}{c}
\omega_{1} \\
\omega_{2} \\
\vdots \\
\omega_{n}
\end{array}\right] \quad \Rightarrow \quad \overrightarrow{\tilde{\omega}}=\left[\begin{array}{c}
\tilde{\omega}_{1} \\
\tilde{\omega}_{2} \\
\vdots \\
\tilde{\omega_{n}}
\end{array}\right] \quad \Rightarrow \quad \vec{\sigma}=\left[\begin{array}{c}
\sigma_{1} \\
\sigma_{2} \\
\vdots \\
\sigma_{n}
\end{array}\right] .
$$

It follows that the $\alpha$ and $\beta$ terms can be found by performing a linear fit of the set $[\vec{\omega}, \overrightarrow{\tilde{\omega}}]$. The slope of the resulting best-fit line corresponds to $\alpha$ and the $y$ intercept to $\beta$. Note that $\beta$ is assumed to be slowly varying, and thus remains constant for a particular experiment. This is a valid assumption since bias drift is generally no more than a few degrees per hour, and the experiment lasts for only minutes. ${ }^{10}$ The mean value of $\vec{\sigma}$ is calculated to provide some measure of the standard deviation of the system for a particular test. However, in practice, standard deviations are higher at larger angular rates due to vibrations associated with the rate table.

To visualize change in calibration one can simply plot $\tilde{\vec{\omega}}$ vs. $\vec{\omega}$. A well calibrated unit would produce a line of the form $y=x$. Changes in calibration parameters are visually apparent when the y intercept becomes non-zero or the slope differs from one. Basic schematic examples are shown in Figure 1.

\section{Allan Variance and Angular Random Walk}

Allan variance $\left(\sigma_{A}^{2}\right)$ is a statistical property calculated from a long data set produced while a sensor is at rest. ${ }^{11}$ It is a function of some time constant, $\tau$, which is also referred to as bin size. To calculate $\sigma_{A}^{2}$, the data set is split into bins of size $\tau$ and the average value within each bin found. Then the following equation can be applied:

$$
\sigma_{A}^{2}(\tau)=\frac{1}{2(n-1)} \sum_{k=2}^{n}\left(y(\tau)_{k}-y(\tau)_{k-1}\right)^{2} .
$$




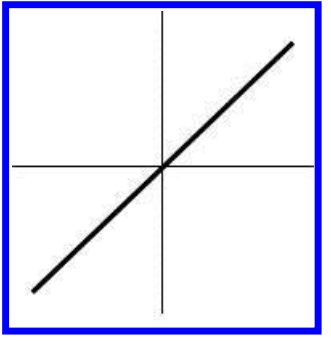

(a)

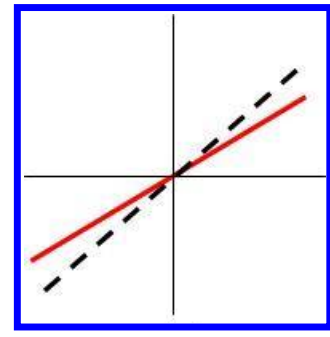

(b)

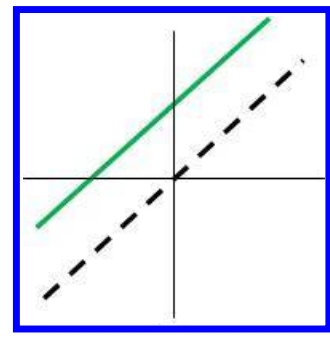

(c)

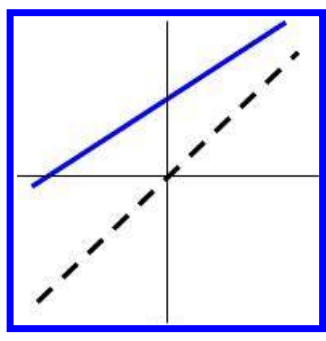

(d)

Figure 1. Schematic examples of change in calibration parameters as seen in a plot of measured vs. actual angular speed. (a) Desired behavior of form $y=x$ (b) Decrease in scale factor (c) Increase in zero offset bias (d) Decrease and scale factor with an increase in bias

In this equation, $y(\tau)_{k}$ is the average value of the $k^{t h}$ bin of size $\tau$, and $n$ is the total number of bins. When the root of the Allan variance, also called the Allan deviation, is calculated over a range of $\tau$ and plotted on a log-log scale, an Allan variance plot is created. A typical example produced from experimental data is given in Figure 2. This plot provides an objective measure of the amount and type of noise in a system and important values such as angular random walk (ARW). In simple terms, ARW describes the amount of white noise in a signal independent of other characteristics such as bias. ${ }^{12}$ The term relates to the error in an angular position calculation made by integrating a rate gyro measurement. To determine ARW from an Allan variance plot, one must find where a tangent line of slope -0.5 intersects the vertical line of $\tau=1$ as shown in Figure 3. A method to calculate ARW using Allan variance was developed for this experiment; however, details are outside the scope of this paper. A complete development of Allan Variance and how it relates to ARW is available in the literature. ${ }^{13-15}$

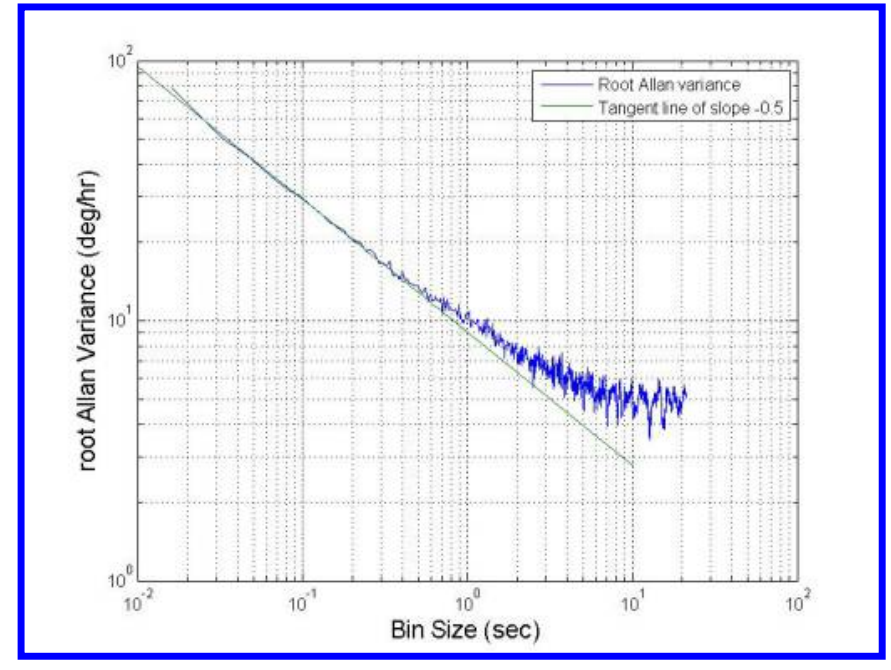

Figure 2. Sample plot of the root Allan variance as a function of the bin size, $\tau$.

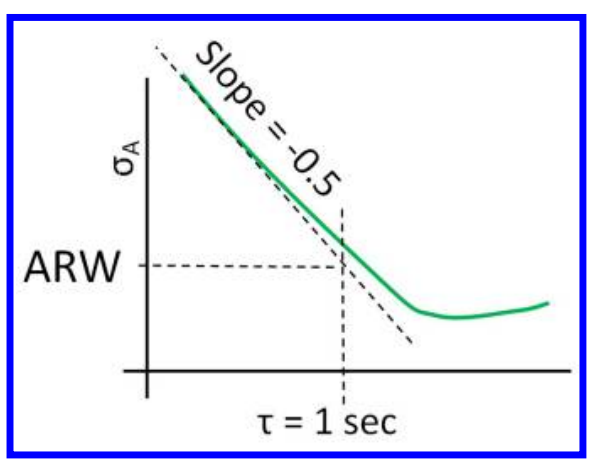

Figure 3. Schematic of how to find ARW from an Allan variance plot.

\section{Helium Exposure Testing}

This section explores the effect that exposure to a helium environment has on the long term operation of the tested gyro units. The general experimental procedure is described and then results using the parameters discussed in Section II are presented. 


\section{A. Procedure}

The experimental procedure for each unit noted in Section I was similar in that they were all exposed to a high concentration of helium for up to 24 hours. This time was chosen because it is analogous to what a satellite might experience aboard its launch vehicle. After exposure, the units were stored in an atmospheric environment. The performance of each unit was tracked over a period of time to simulate the length of a typical CubeSat mission. The SCR1100 unit was the first to be tested and was exposed to helium for 17 hours on August 27th, 2013. The unit was simply inserted into a helium filled latex balloon for the prescribed amount of time. Its performance was checked every one to two weeks for approximately five months. Before the experiment was terminated, it was exposed to a vacuum of quality $5 \cdot 10^{-4}$ Torr for three hours to determine if such a scenario would result in desirable performance.

The SCR1100 was tested using a rate table to apply a known series of rotations of magnitudes $\pm[1,2$, $4,7,10,20,30,40,50,60] \frac{\mathrm{deg}}{\mathrm{sec}}$ for 30 seconds each. A MATLAB script was used to set the command speed of the rate table for the duration of the test. Data was logged from the unit and later parsed and analyzed in MATLAB.

The L3G and Epson units were soaked and tested in parallel. Both units were exposed to helium for 24 hours on August 27th, 2014. The experiment was terminated after a month and a half because both units exhibited stable outputs by this time. As opposed to using a latex balloon, a multi-layer bladder was created using sheets of polyethylene plastic. This was done due to the difficulty of inserting breakout boards through the neck of a latex balloon. The units were also placed on a foam platform about 1.5" high while in the bladder. Thus, as air diffused into the bladder and settled on the bottom, the sensors remained exposed to a richer helium environment. After inserting the units into the bladder, it was filled with helium and sealed shut. The units were then removed from the helium environment after the prescribed amount of time. Figure 4 shows an image of the inflated bladder as well as a schematic of its construction. Both units were calibrated before the helium exposure occurred to provide a basis for comparison and were tested using the rate table at 1-2 week intervals after the soak. The rates used to test these units were $\pm[0,5,10,20,30$, 60 , and 100] $\frac{d e g}{s e c}$, each applied for 30 seconds. Tests were repeated for each principle axis of each unit. In addition to the primary test, the L3G unit was exposed to helium a second time. This second exposure was done on day 54 of the experiment to determine if a second exposure would further affect the measured parameters.
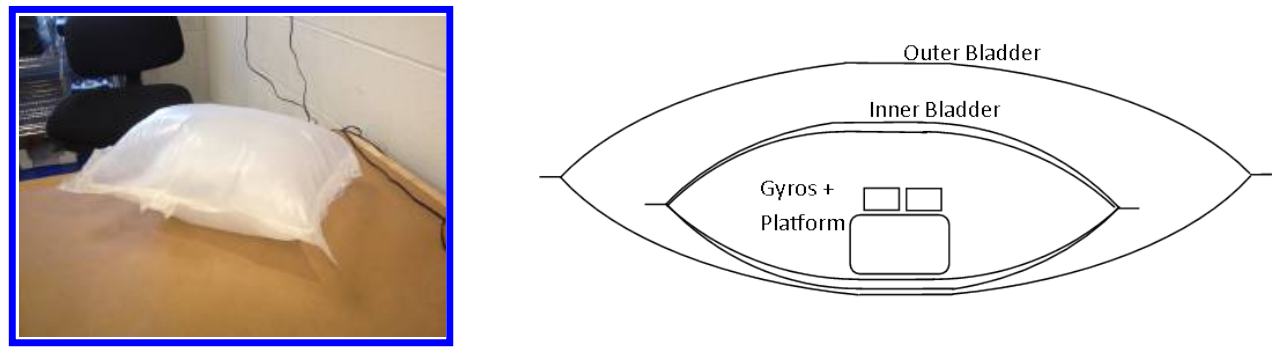

Figure 4. (left) Image of the L3G and Epson units sealed inside of a helium filled plastic bladder. (right) Schematic of the internal structure of the plastic bladder.

Data was collected from the L3G unit using an MSP430 processor connected to an SD card logger. The Epson was connected to a computer using available pass-throughs and data was logged with manufacturer provided software. ${ }^{16}$ The actual rotation rate was measured by polling sensors available within the rate table. Inspection of these measurements revealed that the actual rotation rate had a standard deviation of only $0.05 \frac{\mathrm{deg}}{\mathrm{sec}}$ about the expected rate. Since this experiment is designed to measure the far greater effects of the helium, for analysis it was assumed that the rate table was spinning precisely at the desired speed.

\section{B. Results}

Of the three units tested, the SCR1100 proved to be the most affected by the helium exposure. Over the first two months of the experiment, the zero offset bias drifted from near zero to around $70^{\circ}$ and the scale factor varied significantly. The average standard deviation of the measurements increased from roughly $0.36 \frac{\mathrm{deg}}{\mathrm{sec}}$ to nearly $30 \frac{\mathrm{deg}}{\mathrm{sec}}$. Interestingly, after day 85 of data collection the standard deviation dropped to less than 1 $\frac{d e g}{s e c}$. Furthermore, the calibration terms began to approach their pre-soak values. As mentioned previously, 
the SCR1100 unit was exposed to a vacuum near the end of the experiment. It was hoped that doing so would result in pre-exposure performance. However, this test did not affect the output characteristics of the unit.

Figure 5(a) depicts how the scale factors of the unit changed over time using the method described in Section B. The plot is accompanied by the average standard deviations over all measurements for a particular test. Day 0 corresponds to the pre-helium soak calibration tests, and the first post-soak measurements were done on day 3 .

The L3G unit was moderately effected by the helium exposure, but not to the same extent as the SCR1100. All three axes of the unit showed some change in behavior. Selected parameters at important times during the experiment are shown in Table 1. Transient behavior was observed, but a steady state was arrived at after approximately four days. Although there are differences between the three principle axes, the scale factor generally increased by $30-40 \%$ after the helium soak. The $x$ and $y$ offset bias increased from near 0 to around $-40^{\circ}$ and $-60^{\circ}$ respectively, while the $z$ bias hovered around zero. The unit was exposed to helium a second time for 24 hours on day 57 of the experiment. This caused a new change in the calibration parameters which reached a steady state after a short transient period. Similar percent changes in scale factor and zero offset bias were observed. Figure 5(b) depicts this information graphically. One can see that the various traces generally lie in two distinct groups. These correspond to the measurements before and after the second soak. The standard deviations remained in the range of $0.5-0.75 \frac{\mathrm{deg}}{\mathrm{sec}}$ throughout the experiment. Small peaks in standard deviation appear to have occurred near the times of the helium soaks. It should be noted that despite the change in calibration parameters, this experiment proved that the L3G units could still be used after exposure to helium due to the relatively stable long term outputs.

Table 1. Selected parameters for the helium soaked L3G unit.

\begin{tabular}{|l|cc|cc|cc|}
\multicolumn{2}{c}{} & \multicolumn{2}{c}{$\mathbf{Y}$} & \multicolumn{2}{c|}{$\mathbf{Z}$} \\
\hline Time [days] & $\boldsymbol{\alpha}$ & $\boldsymbol{\beta}$ & $\boldsymbol{\alpha}$ & $\boldsymbol{\beta}$ & $\boldsymbol{\alpha}$ & $\boldsymbol{\beta}$ \\
\hline 0 (pre-soak) & 0.071 & -0.245 & 0.068 & -2.568 & 0.073 & -0.3758 \\
\hline 3 (post-soak) & 0.080 & -30.243 & 0.078 & -48.135 & 0.079 & -0.723 \\
\hline 4 & 0.095 & -42.341 & 0.092 & -69.107 & 0.095 & 0.300 \\
\hline 56 & 0.089 & -37.661 & 0.087 & -60.883 & 0.090 & -0.006 \\
\hline 58 (second soak) & 0.126 & -67.700 & 0.124 & -114.368 & 0.126 & 2.740 \\
\hline 100 & 0.135 & -74.630 & 0.132 & -126.730 & 0.135 & 3.517 \\
\hline
\end{tabular}

Finally, the Epson unit showed no change in calibration or standard deviation throughout the experiment. Selected parameters are given in Table 2. The scale factor remained at a constant data-sheet value of $0.0125 \frac{\mathrm{deg}}{\mathrm{sec}}$ while the bias drifted slightly, but not outside what would normally be expected given the test conditions. The stability of the unit can be seen graphically in Figure 5(c), which also indicates that the standard deviation remained under $0.5^{\circ}$ for the duration of the experiment. To provide further evidence of the stability of the unit, the angular random walk of the $z$ axis was calculated before the soak occurred and at the end of the experiment. The ARW measurements were 0.21 and $0.16 \frac{d e g}{\sqrt{h r}}$ for both cases respectively. Although these results vary by $25 \%$, this level of inconsistency is not altogether unexpected given the precision of the experiment. A more accurate measurement could be made by isolating the unit from external vibrations. Note that ARW measurements of the L3G sensors are not proved since consistent results were not able to be arrived at even under control conditions. The overall stability of the Epson compared to the other units is attributed to the fact that the gyroscope is hermetically sealed.

Table 2. Selected parameters for the helium soaked Epson unit.

\begin{tabular}{|l|cc|cc|cc|}
\multicolumn{2}{c}{$\mathbf{X}$} & \multicolumn{2}{c}{$\mathbf{Y}$} & \multicolumn{2}{c|}{$\mathbf{Z}$} \\
\hline Time [days] & $\boldsymbol{\alpha}$ & $\boldsymbol{\beta}$ & $\boldsymbol{\alpha}$ & $\boldsymbol{\beta}$ & $\boldsymbol{\alpha}$ & $\boldsymbol{\beta}$ \\
\hline 0 (pre-soak) & 0.0125 & 0.106 & 0.0125 & -0.034 & 0.0125 & -0.100 \\
\hline 3 (post-soak) & 0.0125 & 0.109 & 0.0125 & -0.044 & 0.0125 & -0.098 \\
\hline 53 & 0.0125 & 0.005 & 0.0125 & -0.0182 & 0.0125 & -0.004 \\
\hline
\end{tabular}


Three axis accelerometer data from the Epson was collected alongside the gyro measurements and has also been analyzed. It should be noted that while the Epson gyro is hermetically sealed, the accelerometer is not. Data for this analysis was extracted from four of the rate table tests and two steady-state tests performed to calculate the ARW. In all cases, the unit was stationary for the duration of the analysis. The rate table tests include a calibration done on day 0 and three post soak tests on days 3,8 and 18 while the steady-state tests were performed on days 0 and 56. To account for misalignment of the unit, the euclidean norm of the three primary axis measurements was taken for each sample time. The mean and standard deviation, $\mu_{a}$ and $\sigma_{a}$, of the norm is consequently used for analysis.

Between the various tests $\mu_{a}$ changed little; however, $\sigma_{a}$ showed significant discrepancies. For instance, each test resulted in mean values near $1007.2 \mathrm{mG}$, but $\sigma_{a}$ started at $2.296 \mathrm{mG}$ and trended downward until the final test had a standard deviation of only $0.725 \mathrm{mG}$. The values for mean and standard deviation for each test are given in Table 3. Although all tests did result in consistent average accelerations, the apparently decreasing noise in the data leads to a somewhat curious result in that one would expect the noise to increase. Possible sources of the trend could be effects from the helium or other factors such as inconsistent testing procedures or simply environmental effects. Since the data used to perform this analysis is a by-product of the gyroscope testing, further testing designed specifically to study the accelerometer is needed to come to a conclusive result. Potential improvements include collecting more test data and implementing better procedures to minimize outside factors.

Table 3. Mean and standard deviation values associated with the Epson accelerometer.

\begin{tabular}{|l|c|c|}
\hline Time [days] & $\boldsymbol{\mu}_{\boldsymbol{a}}[\mathrm{mG}]$ & $\boldsymbol{\sigma}_{\boldsymbol{a}}[\mathbf{m G}]$ \\
\hline 0 (pre-soak) $^{\text {par }}$ & 1007.9 & 2.296 \\
\hline 0 (pre-soak) $^{*}$ & 1008.3 & 2.495 \\
\hline 3 (post-soak) $^{*}$ & 1007.2 & 1.527 \\
\hline $8^{*}$ & 1007.2 & 1.581 \\
\hline $18^{*}$ & 1007.6 & 1.217 \\
\hline 56 & 1006.8 & 0.725 \\
\hline
\end{tabular}

* Data was extracted from rate table tests

\section{Epoxy Seal Test}

This section details an attempt to prevent the degradation in gyro performance seen in Section III by encasing an L3G gyro units in a layer of epoxy. Similarly to the prior section, the general procedure is presented and results are discussed.

\section{A. Procedure}

An effort to protect the gyros from the helium environment was undertaken after the detrimental effect of the helium was observed on multiple units. It was decided to simply encase the unit in epoxy due to simplicity, ease of application, and affordability. H72 epoxy, developed by Epoxy Technology ${ }^{17}$ was chosen due to its very low permeability to helium compared to other epoxies as tested by Gerlach, et al. ${ }^{18}$ It should be noted that most CubeSat missions generally use low cost components and can not afford higher quality hermetically sealed sensors. Therefore, the development of a low-cost alternative could lead to significant cost savings.

A mold was developed which allowed for a consistent epoxy application thickness of around 3/16". Furthermore, the mold permits the epoxy to cover both sides of the PCB chip that the gyro is mounted to. This was done due to concerns that the helium might diffuse easily through the PCB material. A number of tests were done to refine the application process and produce a workable mold geometry. To perform the tests, a blank piece of FR4 material was substituted for the electronics chip. Multiple mold geometries were iterated on until one was produced that could be easily filled with epoxy, broken open, and lined with a protective coating. The coating is necessary due to interactions between the epoxy and plastic parts. Both a section view and complete view of the final mold are given in Figure 6. Note that the PCB board is inserted through the slot in the middle of the mold. This was done so the mold could be easily broken and the cast 
L3G Helium Soak Results
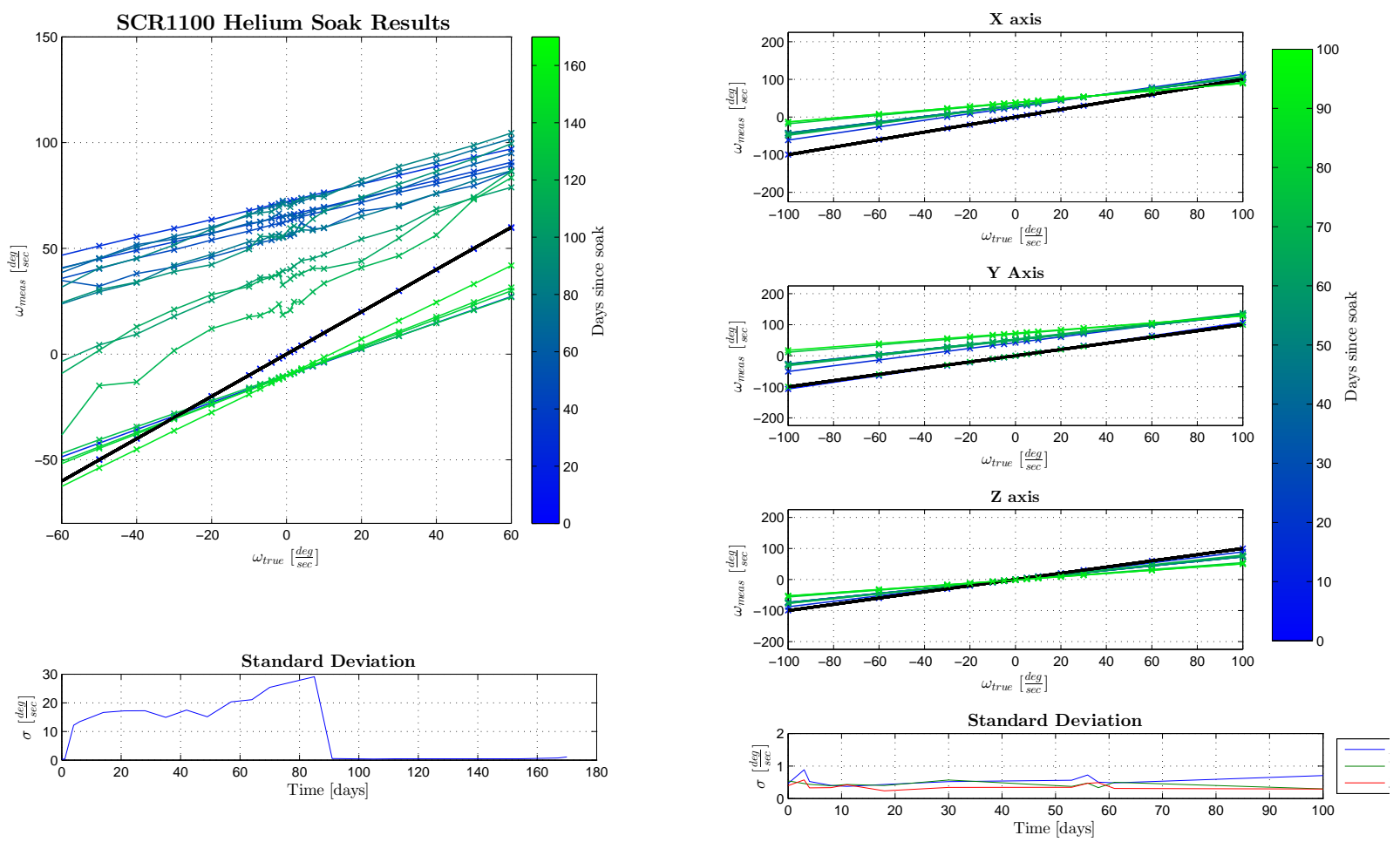

(a)

(b)

Epson MG350 Helium Soak Results
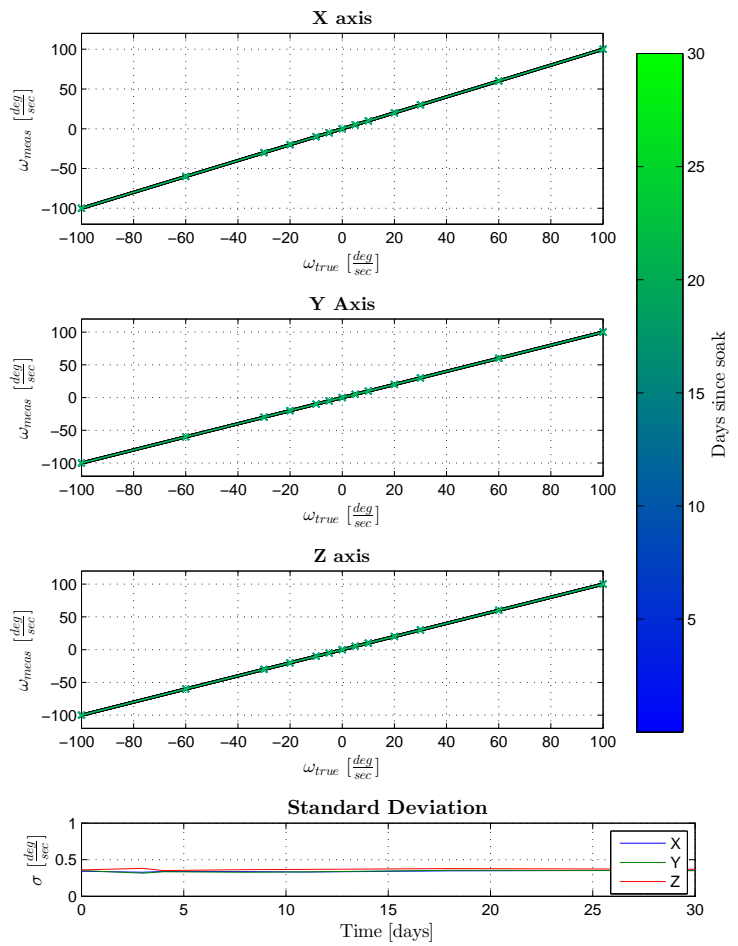

(c)

Figure 5. Set of results from helium exposure testing: (a) SCR1100 (b) L3G (c) Epson.

$$
7 \text { of } 11
$$


retrieved. Otherwise, the mold would need to be cut from the epoxy cast. Angled surfaces are incorporated to aid in the mold release. An overflow region is included on the top to prevent epoxy from spilling over while being de-gassed.
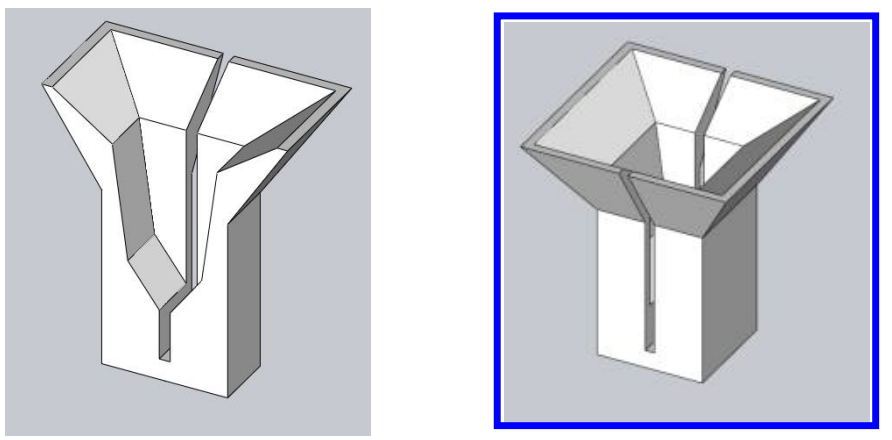

Figure 6. A section view (left) and complete view (right) of the final 3D printed epoxy mold iteration.

The mold itself was manufactured using a MakerBot 3D printer. Other methods, such as using a CNC machine to mill a mold out of wax, were considered. However, the proper tooling for this and other potentially more desirable methods was not available. During testing, it was found that the epoxy chemically reacted with the plastic mold while curing. This led to bubbles forming on the surface of the epoxy which could potentially lead to uncontrolled and undesirable effects. Hence, the inside of the mold was coated with Teflon tape to both protect the plastic mold and act as a release agent. Multiple other coatings, including sheet plastic, turtle wax, and Kapton tape, were tested. The results of some of these tests are shown in Figure 7. The final coated units, along with an uncoated board for comparison, are shown in Figure 8.

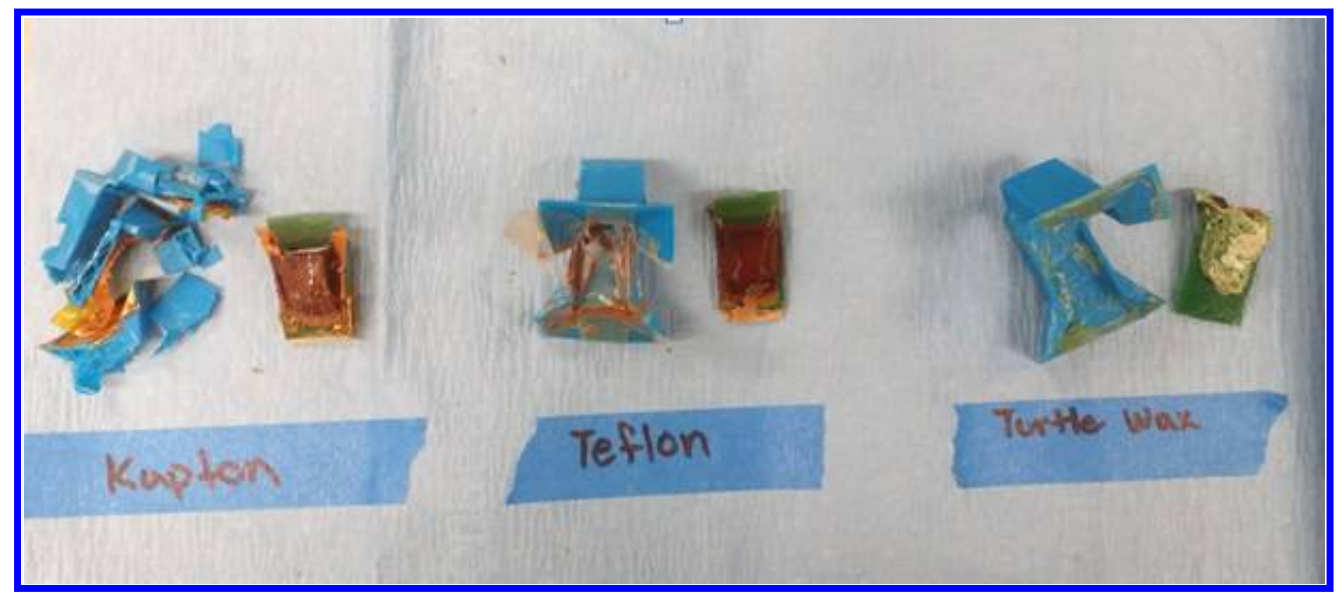

Figure 7. Sample results from three separate mold coating tests. From left to right: Kapton tape, Teflon tape, turtle wax.
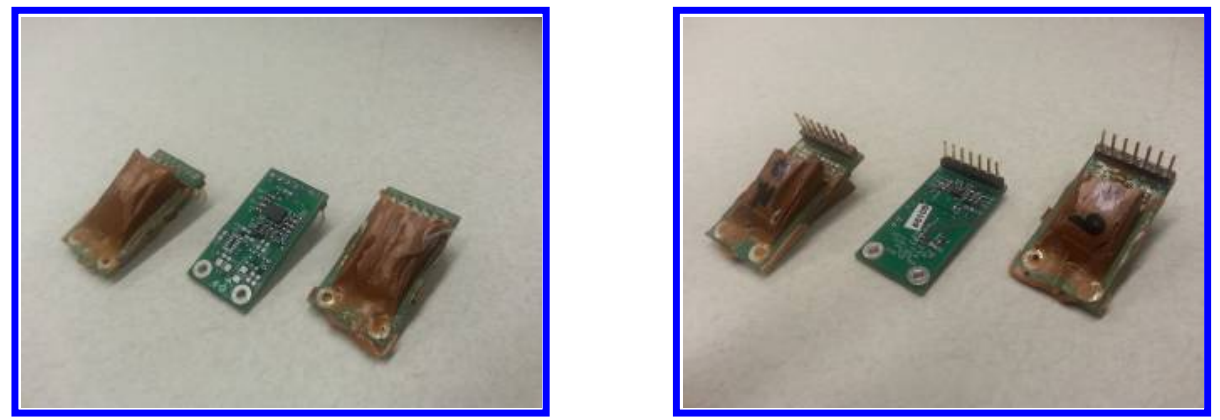

Figure 8. The front (left image) and back (right image) sides of the tested units compared with an unmodified PCB board. The gyroscope itself is the rectangular black chip on the front of the PCB near the pins. 
Although it was intended that the epoxy encapsulate both sides of the chip, the back side of the PCB was only partially covered. This was a result of the placement of the breakout pins on the board. These pins were covered with soft foam when the board was inserted into the mold so they would not incidentally be coated in epoxy. When the epoxy was degassed it expanded around the foam, but was too viscous to flow back into the mold. Thus, the back of the board was left partially uncovered.

The epoxy application process proceeded as follows:

1. Coat the inside of the mold using adhesive backed Teflon tape.

2. Insert the breakout board into the mold and place foam over the pins to ensure they are not coated in epoxy. Place the mold in a vice to prevent epoxy cannot escape through the slot where the PCB and plastic mold interface.

3. Mix the two part epoxy as described by the manufacturer.

4. Fill the mold to a depth such that the gyroscope is entirely submerged

5. Place the test sample into a vacuum chamber with sufficiently low pressure to de-gas the epoxy.

6. Remove sample from chamber and place into an oven at $100^{\circ} \mathrm{C}$ for 30 minutes. This curing leads to the maximum amount of cross-linking for the H72 epoxy which is advantageous to prevent diffusion. ${ }^{18}$

7. Remove from oven and let cool to room temperature.

8. Break the mold off the epoxy cast.

The units were tested once before being coated in epoxy, and again after the epoxy application but before being exposed to helium. The epoxy was applied on Nov, 14th 2014 and the units were exposed to helium approximately a week later.

\section{B. Results}

The epoxy encapsulation tests were done on two separate L3G units, denoted unit 'A' and 'B'. Selected tabulated parameters for the two units are given in Tables 4 and 5 as well as plots in Figure 9. In both cases, day 0 refers to the calibration done before the epoxy coating, day 7 corresponds to the set of measurements taken immediately following the epoxy application, and the helium soak was performed on day 14 . The general trends in the results are similar so they will not be considered separately.

The results show that the epoxy was generally successful in preventing any long term undesired behavior. There was little difference in the data taken immediately before and after the helium soak occurred. The change in scale factor was on the order of one or two thousandths of a $\frac{d e g}{h r}$ and no significant changes in bias were noted. However, both gyros exhibited interesting short term behavior immediately after the epoxy was applied. In the test done following the epoxy application, the calibration curves appear to have become non-linear. This can be seen as the stray blue lines in Figure 9. However, both units returned to near original states by the time another test was performed 3 days later. The final scale factors were within $15 \%$ of the original values and only minor changes in offset bias were recorded. These differences appear to be attributable to the epoxy application and not the helium itself. The cause of this behavior is unknown. Hypotheses include short term damage done by the corrosive effects of the epoxy, out-gassing of the epoxy as it cures, or potentially residual heat trapped inside the unit.

Table 4. Selected parameters for the epoxy sealed L3G unit A

\begin{tabular}{|l|cc|cc|cc|}
\multicolumn{2}{c}{ X } & \multicolumn{2}{c}{ Y } & \multicolumn{2}{c|}{ Z } \\
\hline Time [days] & $\boldsymbol{\alpha}$ & $\boldsymbol{\beta}$ & $\boldsymbol{\alpha}$ & $\boldsymbol{\beta}$ & $\boldsymbol{\alpha}$ & $\boldsymbol{\beta}$ \\
\hline 0 (pre-epoxy) & 0.070 & 0.966 & 0.067 & -0.807 & 0.069 & -0.885 \\
\hline 7 (post-epoxy) & 0.042 & 0.085 & 0.034 & -0.465 & 0.035 & -0.360 \\
\hline 13 (pre-soak) & 0.080 & -0.094 & 0.065 & -0.769 & 0.067 & -0.869 \\
\hline 26 (post-soak) & 0.081 & -0.750 & 0.067 & 0.832 & 0.068 & -6.813 \\
\hline
\end{tabular}


Table 5. Selected parameters for the epoxy sealed L3G unit B

\begin{tabular}{|l|cc|cc|cc|}
\multicolumn{2}{c}{ X } & \multicolumn{2}{c}{$\mathbf{Y}$} & \multicolumn{2}{c|}{$\mathbf{Z}$} \\
\hline Time [days] & $\boldsymbol{\alpha}$ & $\boldsymbol{\beta}$ & $\boldsymbol{\alpha}$ & $\boldsymbol{\beta}$ & $\boldsymbol{\alpha}$ & $\boldsymbol{\beta}$ \\
\hline 0 (pre-epoxy) & 0.070 & 0.783 & 0.070 & 0.965 & 0.070 & -0.342 \\
\hline 7 (post-epoxy) & 0.036 & 0.526 & 0.036 & 0.337 & 0.036 & 0.526 \\
\hline 13 (pre-soak) & 0.078 & 0.029 & 0.070 & 0.598 & 0.070 & 0.923 \\
\hline 26 (post-soak) & 0.080 & .710 & 0.071 & 10.360 & 0.071 & -3.162 \\
\hline
\end{tabular}

L3G(A) Epoxy Encapsulation Results

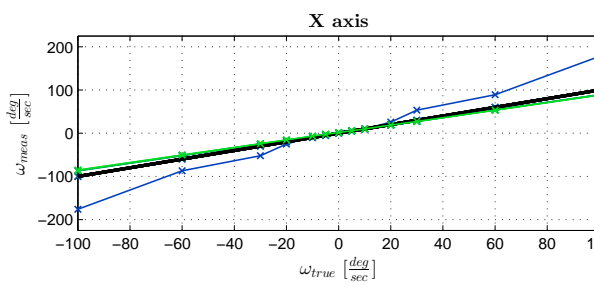

Y Axis

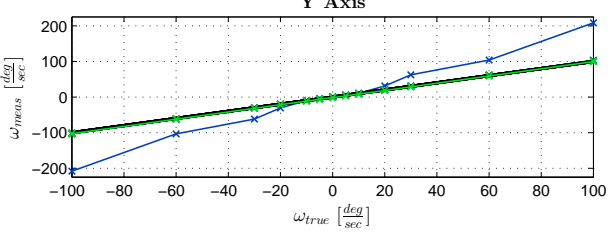

$\mathrm{Z}$ axis
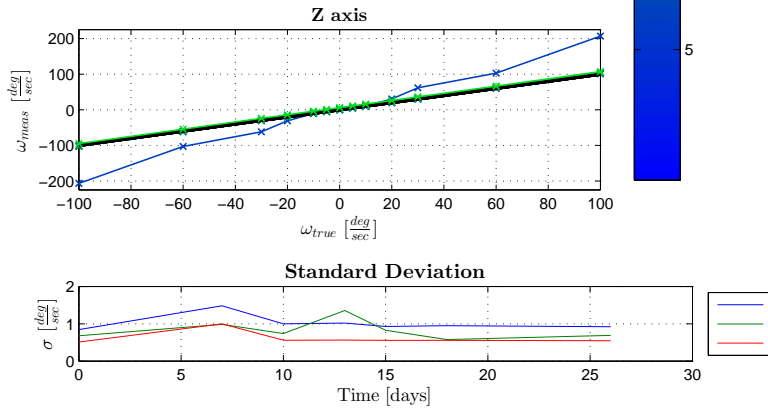

(a)
L3G(B) Epoxy Encapsulation Results

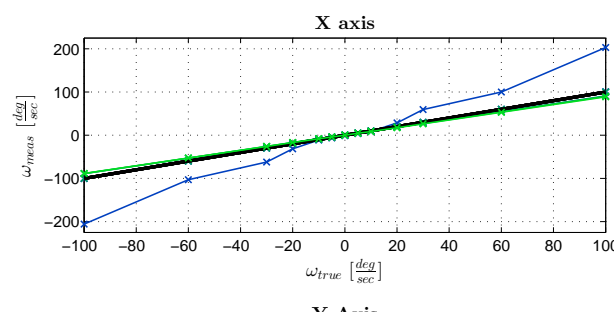

Y Axis

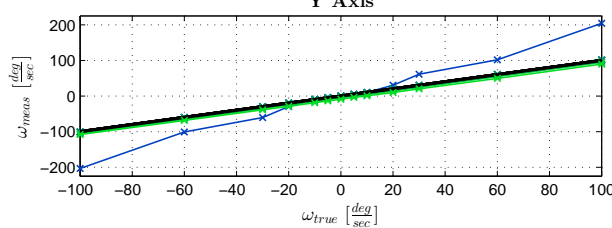

$\mathrm{Z}$ axis

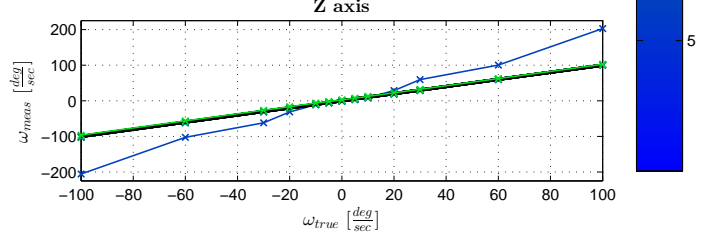

Standard Deviation

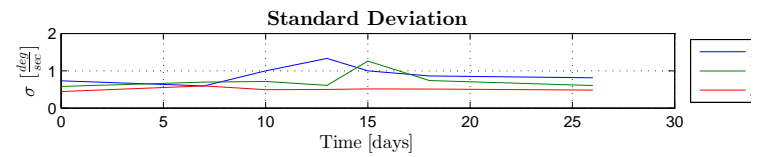

(b)

Figure 9. Set of results from the epoxy encapsulation tests for L3G units (a) A and (b) B.

\section{Conclusions}

In this paper, the effect of helium exposure on MEMS devices is explored and an attempt is made to prevent negative side effects. An overview of analysis tools used to calculate scale factor, bias, and angular random walk is provided. The experiments showed that exposure to helium can significantly affect the operation of inertial devices. Three different gyroscope models, a muRata SCR1100, ST L3G4200D, and Epson M-G350 were tested. Significant undesired effects were observed on the SCR1100 while the L3G4200D faired much better in that the calibration parameters reached steady values following an initial transient period. The Epson was essentially unaffected by the exposure due to a hermetically sealed case. An analysis of the Epson accelerometer data appears to be favorable, but more testing needs to be done to confirm the finding. A second helium soak of the L3G unit resulted in additional variation of parameters. Finally, epoxy applied to two L3G units proved to be an effective barrier to helium; however, the application of the epoxy itself had an undesirable effect on the output of the unit. 
There are numerous avenues for future work based on this project. First, more testing should be done on the MEMS sensors presented in this paper as well as others to determine the consistency of failures. It should be noted that the most effective way to deal with helium exposure is to invest in high quality sensors that are hermetically sealed. However, these devices can be prohibitively expensive considering most CubeSats are low cost and built on affordable components. Therefore, future work should focus on developing the encapsulation method. Experiments need to be done to determine the minimum amount of epoxy necessary to prevent failure and the molding process must be refined. Importantly, an epoxy application procedure that does not itself damage the sensor must be developed and thoroughly tested. These, as well as other potential practical matters, must be thoroughly tested before any solution is applied to flight hardware.

\section{Acknowledgments}

The authors would like to thank other members of the MXL team whom contributed to this experiment, most notably Alyson Mosher who performed the initial testing of the SCR1100 unit. Furthermore, we thank Raymond Chow and Epson Electronics for providing support and supplying the team with Epson M-G350 test units.

\section{References}

${ }^{1}$ Kin, B., Candler, R., Melamud, R., and et. al., "Hermeticity and diffusion investigation in polysilicon film encapsulation for microelectromechanical systems," Journal of Applied Physics, Vol. 105, 2009.

25parks, D., "The hermeticity of sealed microstructures under low temperature helium and hydrogen exposure," Journal of Micromrchanics and Microengineering, Vol. 23, Hanking Electronics Ltd, 2013.

${ }^{3}$ Sparks, D., Mitchell, J., and Lee, S., "Output drifting of vacuum packaged MEMS sensors due to room temperature helium exposure," Journal of Sensor Technology, Vol. 3, Hanking Electronics Ltd, 2013, pp. 101-109.

4 "AeroCube-OCSD," https://directory.eoportal.org/web/eoportal/satellite-missions/a/aerocube-ocsd, accessed: March 23, 2015.

5 "MCubed-2/COVE," https://directory.eoportal.org/web/eoportal/satellite-missions/m/mcubed-2, accessed: March 23, 2015.

6 "SCR1100-D02 data sheet," http://www.muratamems.fi/sites/default/files/documents/scr1100-d02_datasheet_v2. 0_0.pdf, accessed: August 28th, 2014.

7 "L3G4200D Gyro data sheet," http://www.pololu.com/file/0J491/L3G4200D.pdf, accessed: August 28 th, 2014.

8 "Epson M-G350-PD11 Data Sheet," http://www.epsondevice.com/webapp/docs_ic/DownloadServlet?id=ID002527, accessed: June 18, 2014.

${ }^{9}$ Cutler, J., Ridley, A., and Nicholas, A., "Cubesat Investigating Atmospheric Density Response to Extreme Driving (CADRE)," Small Satellite Conference, 2011.

${ }^{10}$ Springmann, J. C. and et. al., "The attitude determination system of the RAX satellite," Acta Astronautica, Vol. 75, 2012.

${ }^{11}$ Stockwell, W., "Angle Random Walk," Tech. rep., Crossbow Technology.

${ }^{12}$ Stockwell, W., "Bias Stability Measurement: Allan Variance," Tech. rep., Crossbow Technology.

$\checkmark{ }^{13}$ El-Sheimy, N., Hou, H., and Niu, X., "Analysis and modeing of inertial sensors using allan variance," IEEE Transactions on Instrumentation and Measurement, Vol. 57, January 2008, pp. 140-149.

${ }^{14}$ Vagner, M., "MEMS gyroscope performance comparison using allan variance method," 2011, EEICT.

${ }^{15}$ IEEE Aerospace and Electronic Systems Society, IEEE standard specification format guide and test procedure for single axis interferometric fiber optic gyros, 1998, IEEE Std 952-1997.

16 "Epson M-G350-PD11 IMU logging software," http://www5.epsondevice.com/en/sensing_system/tech/soft_manual/, accessed: June 18, 2014.

17 "EPO-TEX H72: Technical Data Sheet," Tech. rep., Epoxy Technology, 2010.

${ }^{18}$ Gerlach, A., Keller, W., Shulz, J., and Schumacher, K., "Gas permeability pf adhesives and their application for hermetic packaging of microcomponents," Microsystems Technologies, Vol. 7, 2001, pp. 17-22. 